\title{
Nicotine and the Central Nervous System: Biobehavioral Effects of Cigarette Smoking
}

\author{
Ovide F. Pomerleau, Ph.D., Ann Arbor, Michigan
}

The effects of nicotine, like those of other drugs with potential for abuse and dependence, are centrally mediated. The impact of nicotine on the central nervous system is neuroregulatory in nature, affecting biochemical and physiological functions in a manner that reinforces drug-taking behavior. Dosedependent neurotransmitter and neuroendocrine effects occur as plasma nicotine levels rise when a cigarette is smoked. Circulating levels of norepinephrine and epinephrine increase, and the bioavailability of dopamine is altered as well. Among the neuroendocrine effects are release of arginine vasopressin, $\beta$ endorphin, adrenocorticotropic hormone, and cortisol. Notably, several of these neurochemicals are psychoactive and/or known to modulate behavior. Thus, affective states or cognitive demands may be favorably modified (at least temporarily) by nicotine intake. When nicotine is inhaled, the neuroregulatory effects just described are immediately available and the reinforcing effects of the drug are maximized. On the other hand, nicotine gum and most other nicotine replacement vehicles in current use have a slower onset of action, resulting in less reinforcement value. Recent data suggest that smoking cessation rates may be optimized by tailoring the dose of nicotine replacement (for example, 2 or $4 \mathrm{mg}$ of nicotine gum) to the individual degree of nicotine dependence. In view of the dynamic interactions between the neuroregulatory effects of nicotine and a host of environmental conditions, nicotine replacement therapy is best carried out in combination with behavior modification techniques.
From the Behavioral Medicine Program, Department of Psychiatry, University of Michigan, Ann Arbor, Michigan.

Requests for reprints should be addressed to Ovide F. Pomerleau, Ph.D., Behavioral Medicine Program, 900 Wall Street, Ann Arbor, Michigan 48105 .
D rug dependence has been defined as $s u b$ stance-seeking behavior involving a psychoactive agent that acts on the central nervous system [1]. All drugs with abuse liability have dose-dependent effects that can be discriminated from those of inactive substances, and these effects are centrally mediated.

In rats, subcutaneous administration of nicotine has been shown to produce dose-dependent increases in cerebral glucose uptake, indicating heightened brain metabolic activity [2]. Similar changes in brain energy utilization have been observed in association with such drugs as cocaine. Central mediation of the effects of nicotine has been demonstrated by administration of mecamylamine, a nicotinic cholinergic blocker that crosses the blood-brain barrier. In contrast to the nicotine antagonist hexamethonium, which acts only peripherally, mecamylamine blocks increases in cerebral glucose uptake and produces some degree of neutralization of the effects of nicotine that are required for maintaining dependence.

Drugs of abuse are characteristically euphoriants; these effects are dose dependent and centrally mediated. In studies of human subjects, nicotine has demonstrated dose-related euphoric effects similar to those of cocaine and morphine [3]. Notably, however, the dose range at which nicotine is effective but not toxic is relatively narrow. This observation has important implications for the management of nicotine dependence.

Nicotine has been shown to be the psychoactive substance in cigarette smoke that accounts for both self-administration and reinforcement of drugtaking behavior. In one study, double-blind substitution of saline for nicotine resulted in discontinuation of self-administered injections by cigarette smokers [3]. Subsequently, when the subjects were allowed to choose between nicotine and placebo, they chose nicotine-demonstrating that nicotine functioned as a positive reinforcer.

Drug dependence may be associated with tolerance and physiologic withdrawal, although current concepts hold that these components are not essential to the definition of dependence [1]. Nicotine withdrawal occurs with abrupt cessation or reduction in nicotine intake in a person who has used the 
drug daily for at least several weeks and is characterized by the appearance within 24 hours of at least four of the following signs $[4]$ :

- nicotine craving;

- irritability, frustration, or anger;

- anxiety;

- difficulty concentrating;

- restlessness;

- decreased heart rate;

- increased appetite.

Nonetheless, apart from the first cigarette of the day or cigarettes smoked after prolonged deprivation, much smoking behavior seems to be prompted by independent environmental cues rather than the need to terminate or avoid withdrawal [5]. The explanation for this behavior may rest in specific, centrally mediated effects of nicotine that allow the smoker to gain temporary improvements in performance or affect.

\section{NEUROREGULATORY EFFECTS OF NICOTINE}

The impact of nicotine on the central nervous system is neuroregulatory in nature, with cascading effects on physiological and biochemical functions. The dose-related effects of nicotine on neurotransmitters and neuroendocrine responses therefore constitute a critical component of its pharmacologic action-effects that are responsible for both reinforcement of nicotine self-administration and pathophysiological consequences.

\section{Effects on Catecholamines}

After a cigarette is smoked, circulating levels of catecholamines increase as plasma nicotine levels rise [5]. (Figure 1 shows the effects of smoking a typical cigarette [approximately $1 \mathrm{mg}$ nicotine content].) Even when there is only a modest increase in plasma nicotine level (as seen in individuals who have already smoked several cigarettes during the day), a profound increase in circulating norepinephrine levels is observed. These increased levels of both nicotine and norepinephrine subsequently decay rapidly. Epinephrine levels rise in a dosedependent fashion when a cigarette is smoked, and nicotine also alters the bioavailability of dopamine. All these effects are most pronounced following the first eigarette of the day.

\section{Effects on Hypophyseal Hormones}

Several studies by my colleagues and myself a decade ago focused on the response of pituitary hormones to various increases in nicotine levels [6-8]. After overnight deprivation, subjects were instructed to smoke either a low-dose cigarette in an ordinary fashion, a high-dose cigarette in an ordi- nary fashion, or two high-dose cigarettes in rapid succession, with deep inhalation (sufficient to produce nausea).

In this series of studies, nicotine levels increased by approximately $5-6 \mathrm{ng} / \mathrm{mL}$ with the low-dose cigarette; by $20-25 \mathrm{ng} / \mathrm{mL}$ with the high-dose cigarette smoked in a normal manner; and by $60 \mathrm{ng} / \mathrm{mL}$ with the high-dose cigarettes smoked deeply $[6,8]$. As shown in Figure 2, dose-related increases were observed in plasma levels of arginine vasopressin and $\beta$-endorphin (although the assay used in this study had $50 \%$ co-reactivity with $\beta$-lipotropin). Animal experiments have shown nicotine dose-related release of endogenous opioids in both hippocampal and hypothalamic sites [9]. In other words, not only do circulating endogenous opioids increase in response to nicotine, but release also occurs centrally.

Dose-related increases in adrenocorticotropic hormone (ACTH) and cortisol were demonstrated as well, as shown in Figure $3[7,8]$. Al the highest doses of nicotine, significant increases were also obscrved in growth hormone and prolactin, but thy-

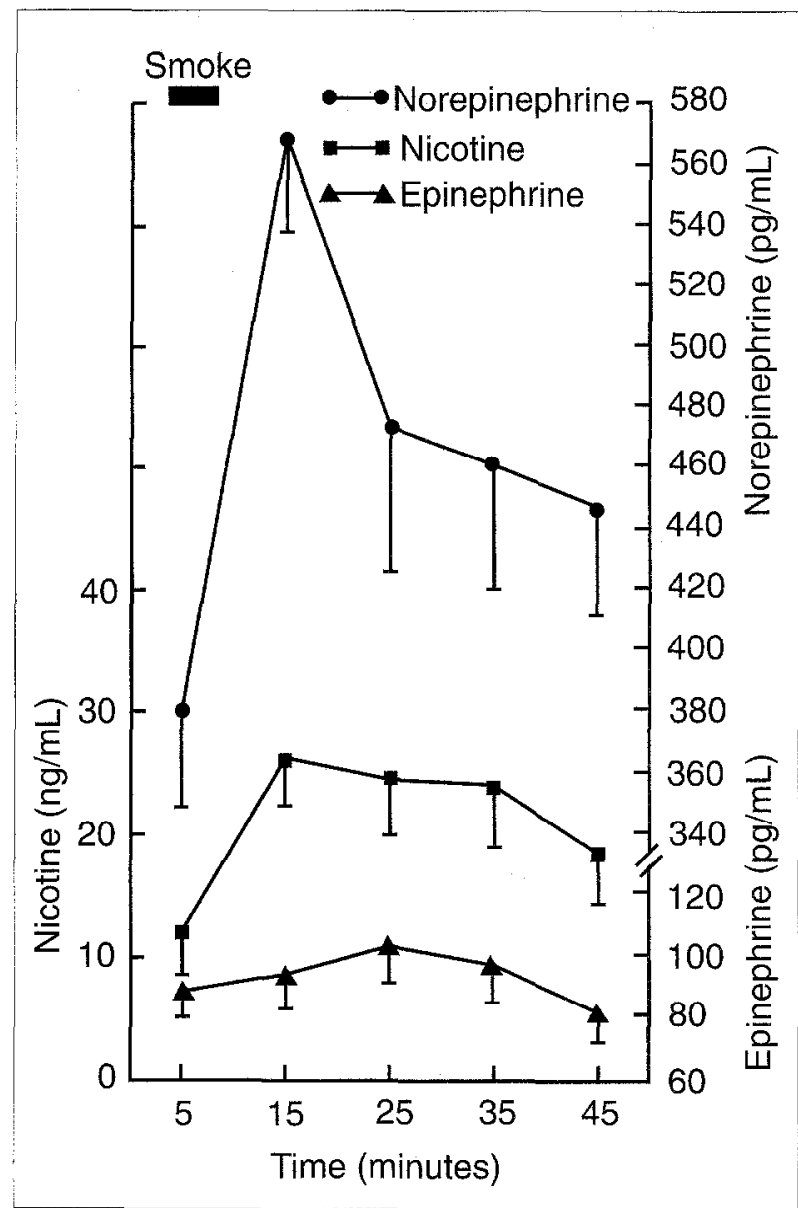

Figure 1. Patterns of change in serum norepinephrine and epinephrine levels relative to nicotine concentrations resulting from a single cigarette (approximately $1 \mathrm{mg}$ nicotine content). Mean ( \pm SEM) for 10 smokers who smoked following a 1.5-hour interval without cigarettes. 
roid-releasing hormone, thyroid-stimulating hormone, gonadotropin-releasing hormone, and various sex hormones were not affected.

\section{BEHAVIORAL IMPLICATIONS}

Several of the neuroregulators that exhibit altered bioavailability in response to nicotine are known to have behavioral manifestations [5]. Con- trol of sympathetic tone via norepinephrine mediation in the locus coeruleus, for example, may facilitate ability to focus and concentrate. Central acetylcholine release has been shown to be involved in memory and task performance, thereby implicating the cholinergic component of the nervous system. In addition to its antidiuretic and vasoconstrictive effects, arginine vasopressin has been associated
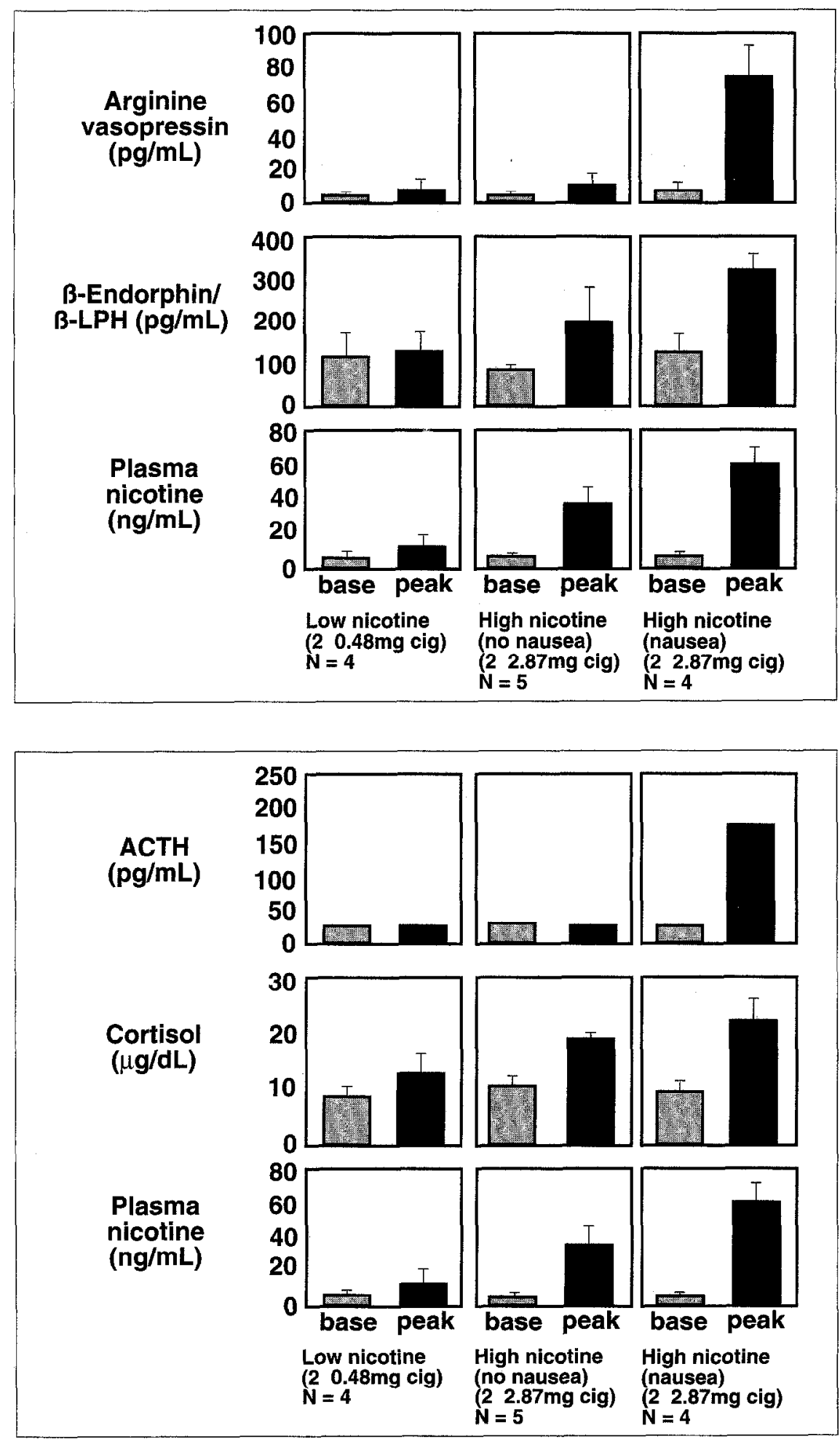

Figure 2. Baseline and peak levels of serum arginine vasopressin and $\beta$-endorphin/ $\beta$-lipotropin in subjects smoking a low-dose cigarette in ordinary fashion, a high-dose cigarette in ordinary fashion, or two high-dose cigarettes in rapid succession, with deep inhalation (sufficient to produce nausea in some cases). $\beta$ - LPH $=$ $\beta$-lipotropin. Adapted with permission from [6] and [8].
Figure 3. Baseline and peak serum levels of adrenocorticotropic hormone and cortisol in subjects smoking a low-dose cigarette in ordinary fashion, a high-dose cigarette in ordinary fashion, or two high-dose cigarettes in rapid succession, with deep inhalation (sufficient to produce nausea in some cases). $\mathrm{ACTH}=$ adrenocorticotropic hormone. Adapted with permission from [7] and [8]. 
with memory consolidation and retrieval. Endogenous opioids, such as $\beta$-endorphin, not only potentiate vagal reflexes, decreasing respiratory rate and blood pressure by opposing the effects of catecholamines, but are also associated with antinociception and anxiety reduction. Finally, the stimulation of cortisol by ACTH has been shown to modulate attention and stimulus discrimination and, in more recent rescarch, to diminish the sensitivity of nicotine receptors to subsequent stimulation.

Examination of the psychological consequences of smoking suggests that affective states or cognitive demands can be modified in a favorable or adaptive manner, at least temporarily, by nicotine stimulation (Table I) [5]. By increasing central dopaminergic turnover, for example, nicotine can elicit or enhance "pleasure." Increases in norepinephrine and $\beta$-endorphin may be implicated in these effects as well.

Task performance may be facilitated by the effects of nicotine on acetylcholine and norepinephrine. Brief improvement in memory recall is likely related to effects on acetylcholine, norepinephrine; and perhaps vasopressin, whereas anxiety and tension may be relieved by increases in $\beta$-endorphin. Dopamine and norepinephrine may be involved in avoidance of weight gain (possibly because of a reduction in hunger), although an increasing body of evidence suggests that serotonin is also involved in these effects [10]. Cholinergic activity mediates relief from nicotine withdrawal, and corticosteroid modulation of nicotinic receptor sensitivity may be involved as well.

\section{THERAPEUTIC INTERVENTIONS}

\section{Role of Nicotine Replacement Therapy}

The administration of nicotine via cigarette smoking is particularly well suited to maximizing the neuroregulatory impact of the drug. Inhaled nicotine undergoes rapid absorption, with approximately $25 \%$ of the inhaled drug reaching the brain within 7 seconds [1]. Nicotine from inhalation reaches the brain twice as fast as from intravenous administration.

Inhaled nicotine produces a sharp rise in serum concentrations, with peak concentrations nearly always achieved by the time an individual has finished smoking a cigarette (Figure 4). Consequently, the reinforcing effects of the drug are immediately available to the smoker and probably do not outlast the circumstances that prompted the smoking. Intranasal administration also results in an abrupt increase in serum concentrations and is currently being investigated as a method of treating nicotine dependence [11].

In contrast to inhaled nicotine, nicotine gum (nic-

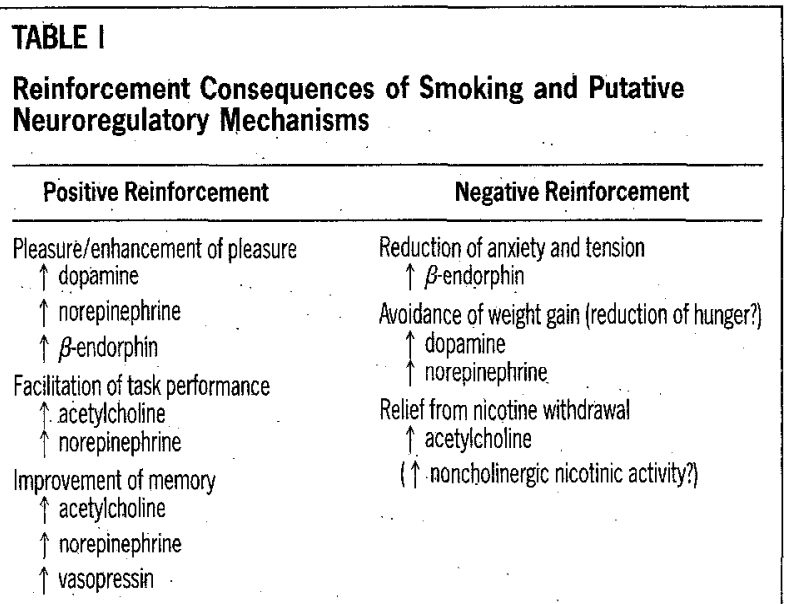

Adapted with permission from [5].

otine polacrilex) has a much slower onset of action, and its effects are sustained well beyond the circumstances prompting nicoline self-administration [12]. Because peak concentrations are reached approximately 0.5 hour after initial administration, less reinforcement value is realized than with cigarettes. Unlike cigarettes, therefore, nicotine gum has a low potential for abuse.

According to one study, as the dose of nicotine gum is elevated, its perceived strength and similarity to cigarettes are increased correspondingly [13]. Since the $4 \mathrm{mg}$ dose is associated with decreased patient acceptance and a higher incidence of side effects, compared with the $2 \mathrm{mg}$ dose, these considerations must be balanced against the greater relief of nicotine craving achieved with the higher dose.

In a recent investigation, degree of nicotine dependence was used to determine which nicotine replacement dose should be administered [14]. More-dependent smokers generally have a poorer prognosis than less-dependent smokers, but when the $4 \mathrm{mg}$ dose was prescribed to high-dependency smokers, smoking cessation rates were comparable to those achieved by low-dependency smokers using the $2 \mathrm{mg}$ dose (Figure 5). Similar findings for transdermal nicotine therapy suggest that the benefits of matching dosage to degree of dependency apply to all nicotine replacement vehicles.

\section{Role of Behavior Modification}

The neuroregulatory effects of nicotine are not fixed, but rather are dynamic-reflecting interactions with environmental conditions, history of nicotine use, and ongoing behavior. A large number of internal and external cues unrelated to nicotine dependence serve to prompt smoking. Consequently, nicotine replacement therapy, which focuses mainly on relief of cigarette withdrawal, is best carried out in conjunction with behavior modi- 
fication in order to strengthen the new habits required to make the transition from smoker to nonsmoker.

In one of several reports on the issue, the use of nicotine gum alone was associated with a smoking cessation rate only slightly higher than that resulting from the use of skills training alone [15]. When the two modalities were combined, however, the smoking cessation rate was better than for either modality in isolation, and the improvement was sustained into the follow-up period 10.5 months after the start of treatment. In general, research has shown that pharmacological intervention is most effective when applied in a context that in-
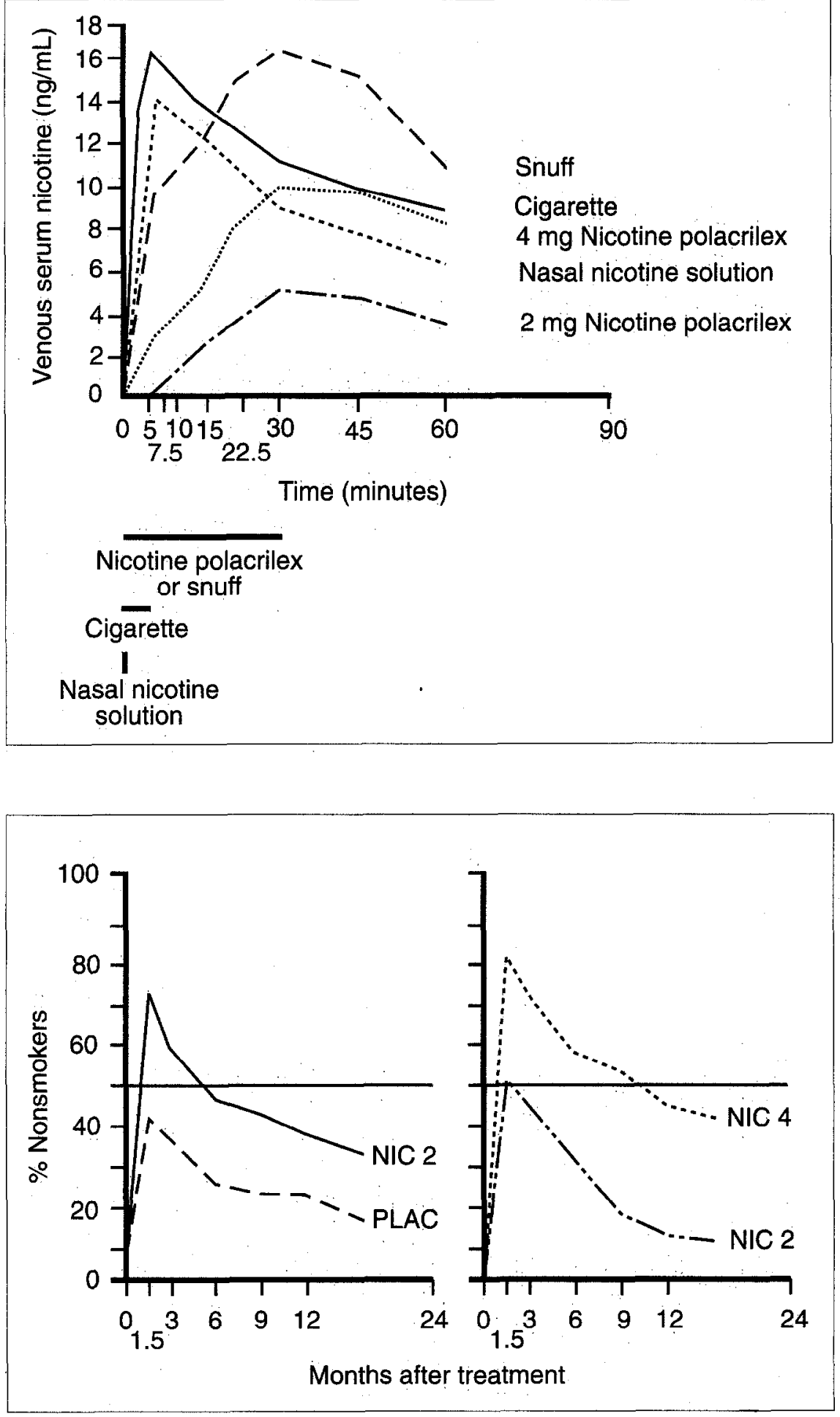

Figure 4. Serum nicotine levels after administration of nicotine via cigarette smoking, via nicotine gum ( 2 or $4 \mathrm{mg}$ nicotine polacrilex), or intranasally. Reprinted with permission from [12].
Figure 5. Smoking cessation rates in lowdependency smokers randomized to receive 2 mg nicotine gum (NIC 2) or placebo (PLAC) versus high-dependency smokers randomized to receive 2 or $4 \mathrm{mg}$ (NIC 2 or 4 ) nicotine gum. Adapted with permission from [14]. 
cludes social support and the inculcation of new skills [1]. Behavioral intervention is particularly useful for increasing adherence to pharmacological treatment.

\section{CONCLUSION}

Much remains to be learned about the neuroregulatory mechanisms by which nicotine exerts its effects. Current knowledge has been largely derived from studies in which nicotine agonists and antagonists have been administered under a variety of environmental conditions [9]. More information is needed on the timing and duration of neuroregulator release as well as the pattern of neuroendocrine responses, relative to the history of nicotine use, varying environmental conditions, and mode of administration. In the future, newer research approaches-e.g., blood flow and metabolic studies using techniques such as positron emission tomography - may shed light on the particular neuroregulatory pathways involved in human smoking. These technologies should facilitate the specification of the neural locus of action of nicotine in relation to its behavioral and physiological effects.

\section{REFERENCES}

1. USDHHS. The health consequences of smoking: nicotine addiction (1988 report of the surgeon general). Rockville, Maryland: Public Health Service, 1988.
2. London ED, Connolly RJ, Szikszay $M$, et al. Distribution of cerebral metabolic effects of nicotine in the rat. Eur J Phamacol 1985; 110: 391-2.

3. Henningfield JE, Miyasato $K$, Jasinski DR. Cigarette smokers self-administer intravenous nicotine. Pharmacol Biochem Behav 1983; 19: 887-90.

4. Diagnostic and Statistical Manual of Mental Disorders, 3rd ed, rev. Washington, D.C.: American Psychiatric Association, 1987.

5. Pomerleau OF, Pomerleau CS. Neuroregulators and the reinforcement of smoking: towards a biobehavioral explanation. Neurosci Biobehav Rev 1984; 8: 503-13. 6. Pomerleau OF, Fertig JB, Seyler $E$, et al. Neuroendocrine reactivity to nicotine in smokers. Psychopharmacology 1983; 81: 61-7.

7. Seyier LE, Fertig J, Pomerleau 0 , et al. The effects of smoking on ACTH and cortisol secretion. Life Sci 1984; 34: 57-65.

8. Seyler LE, Pomerleau OF, Fertig JB, Hunt D, Parker K. Pituitary hormone response to cigarette smoking. Pharmacol Biochem Behav 1986; 24: 159-62.

9. Pomerleau $\mathrm{OF}$, Rosecrans J. Neuroregulatory effects of nicotine. Psychoneuroendocrinology 1989; 14: 407-23.

10. Pomerleau OF, Pomerleau CS, Morrell EM, Lowenbergh JL. Effects of flouxetine upon weight gain and food intake in smokers who reduce nicotine intake. Psychoneuroendocrinology 1991; 16: 433-40.

11. Russell MAH. Nicotine replacement: the role of blood nicotine levels, their rate of change, and nicotine tolerance. In: Pomerleau OF, Pomerleau CS, eds. Nicotine replacement: a critical evaluation. New York: Alan R. Liss, 1988; 63-94. (Paperback edition: Binghamton, New York: Haworth Press, 1992.)

12. Sachs DPL. Nicotine polacrilex: practical use requirements. Curr Pulmonol 1989; 10: 141-58.

13. Henningfield JZE, Jasinski DR. Pharmacologic basis for nicotine replacement. In: Pomerleau CS, eds. Nicotine replacement: a critical evaluation. New York: Alan R. Liss, 1988; 35-61. (Paperback edition: Binghamton, New York: Haworth Press, 1992.)

14. Tonnesen $P$, Fryd $V$, Hansen $M$, et al. Effect of nicotine chewing gum in combination with group counseling on the cessation of smoking. N Engl J Med 1988; 318; $15-8$.

15. Killen JD, Maccoby N, Taylor $\mathrm{CB}$. Nicotine gum and self-regulation training in smoking relapse prevention. Behav Ther 1984; 15: 234-48. 